\title{
Novel Silicon Drift Detector Devices for Ultra-Fast, High-Resolution X-ray Spectroscopy
}

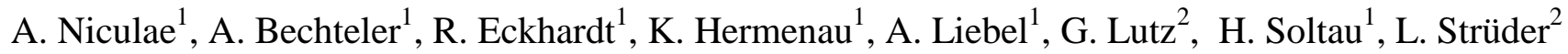 \\ ${ }^{1}$ PNDetector GmbH, Otto-Hahn-Ring 7, 81739 Munich, Germany \\ ${ }^{2}$ PNSensor GmbH, Otto-Hahn-Ring 7, 81739 Munich, Germany
}

Over the last decade the Silicon Drift Detector has become the number one detector choice for many kinds of x-ray spectroscopy applications, especially when high spectral accuracy at high measurement speed is required, as it is often the case in the EDX microanalysis.

With its long experience in the development and manufacturing of the SDD chips and modules, PNDetector is continuously working on the optimization of the detector performance in terms of energy resolution, detection efficiency, detector geometry or solid angle coverage. Owing the unique feature of directly integrating the first FET onto the detector chip which leads to a very small value of the total input capacitance, the energy resolution of the SDD with integrated FET has been pushed continuously towards the theoretical limit set by the statistical fluctuations in the detector material (Fano noise, for silicon: $f w h m \approx 119 \mathrm{eV}$ at $\mathrm{Mn}-\mathrm{K}_{\alpha}$ ).

With the commissioning and the operation of our new detector fabrication line which is dedicated to high-quality, double-sided detector processing, we are in full control of all process parameters leading to ultra-low values of the detector leakage current (e.g. $<80 \mathrm{pA} / \mathrm{cm}^{2}$ at room temperature).

High photon rate applications in microanalysis require an operation of the SDD device at very short processing times while maintaining the superior spectroscopic performance of the detector. This can only be achieved by reducing the total detector capacitance to the lowest possible values. With the introduction of the SDD droplet (SD3) topology combined with the re-design of the detector readout structure including the anode and the integrated FET (SDD ${ }^{\text {plus }}$ FET), the total input capacitance has already been reduced to unprecedented levels down to $50 \mathrm{fF}$ [1]. Optimum energy resolution values down to $122 \mathrm{eV}$ at $1 \mu \mathrm{s}$ shaping time are measured with these devices (see red curve in Figure 2a).

As it can be seen from the black and red plots of Figure 2a, it is the so-called "ballistic deficit" which limits the performance of the standard droplet type SD3 devices at the shortest processing times. Recently we developed a novel SD3 type device which overcomes to a large extent the drawback of the "ballistic deficit" effect by re-designing the entire drift ring structure. Figure 1a shows the drift ring structure of the new SD3 device - which we call SD3 ${ }^{G L}$. For comparison, the drift ring structure of a standard SD3 device is shown in Figure 1b.

The spectroscopic performance of the new SD ${ }^{G L}$ device is shown in Figure 2 a (blue curve). While the measured energy resolution values at optimum shaping times $(1-2 \mu \mathrm{s})$ are very similar to those measured with the SD3 ${ }^{\text {plus }}$ detector, the new device shows clearly better performance at very short shaping time. The energy resolution of $127.5 \mathrm{eV}$ @ $125 \mathrm{~ns}$ shaping time is to our knowledge the best measured value ever reported for with a silicon drift detector.

The new device topology is scalable to larger detector sizes. Figure $2 b$ shows the measured energy resolution of a new $30 \mathrm{~mm}^{2} \mathrm{SD} 3^{G L}$ device as compared to standard $30 \mathrm{~mm}^{2}$ round SDD devices. Further measurements with the new SD3 ${ }^{G L}$ devices will be presented and the results will be discussed.

[1] A. Niculae et al., paper presented at M\&M 2013 in Indianapolis. 


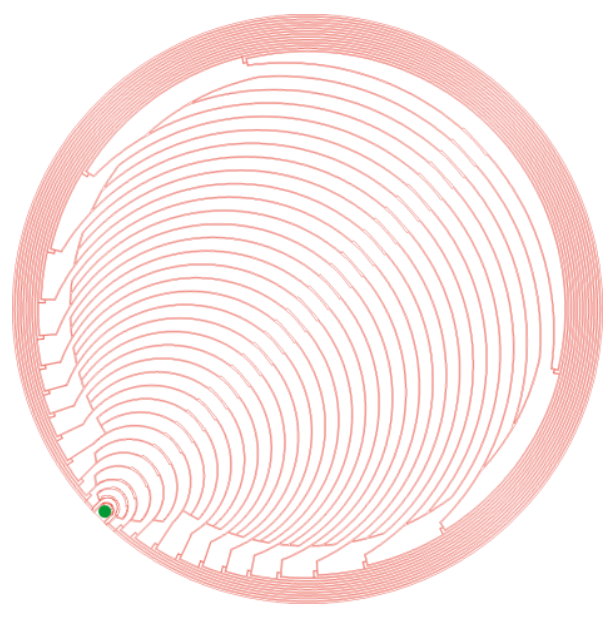

(a)

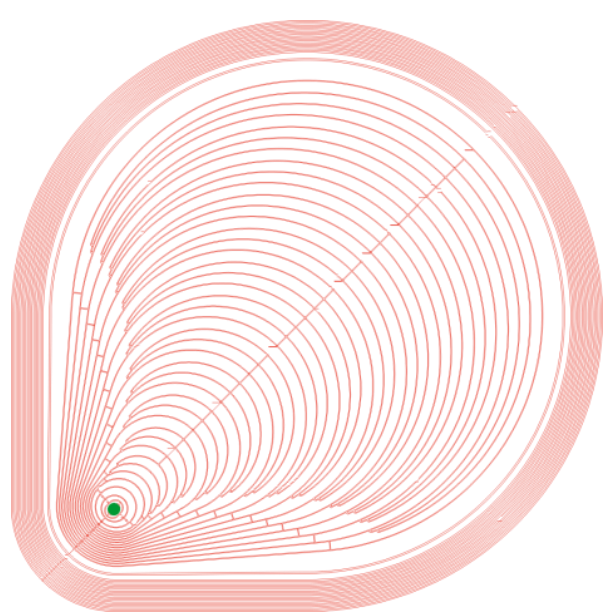

(b)

Fig. 1: (a) View of the drift ring structure of the optimized SD3 ${ }^{G L}$ device. The green dot indicates the readout node including the integrated FET; (b) Drift ring structure of a standard SD3 device.

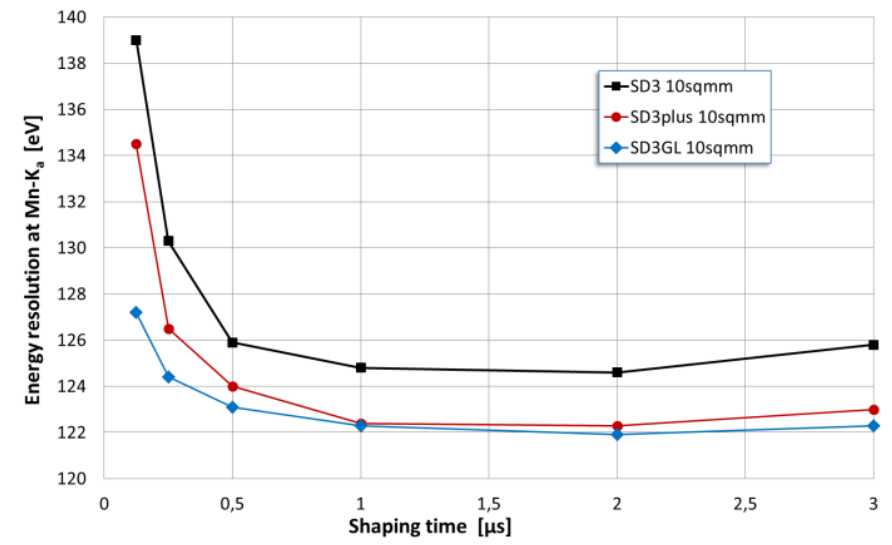

(a)

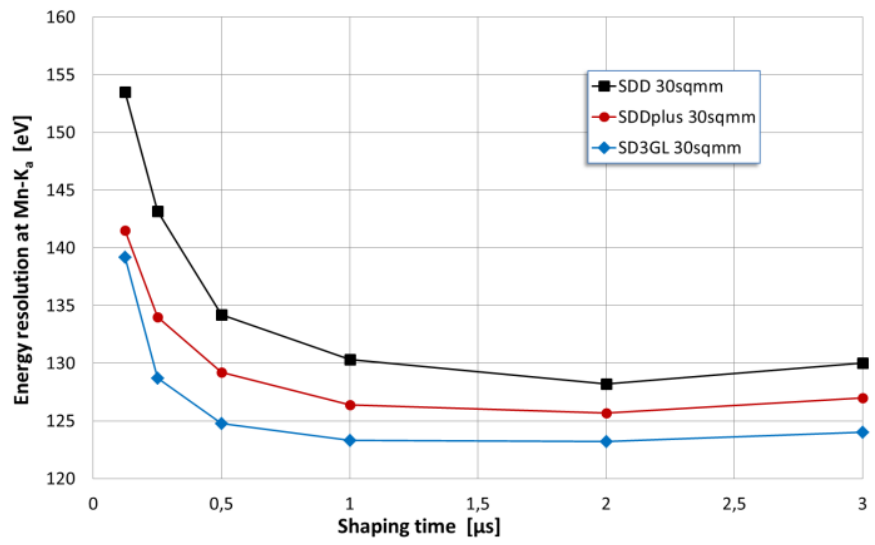

(b)

Fig. 2: (a) Energy resolution versus shaping time for a $10 \mathrm{~mm}^{2} \mathrm{SD} 3^{G L}$ device compared to standard SD3 devices; (b) Energy resolution of a $30 \mathrm{~mm}^{2} \mathrm{SD}^{G L}$ device compared to other $30 \mathrm{~mm}^{2}$ round SDD devices. 